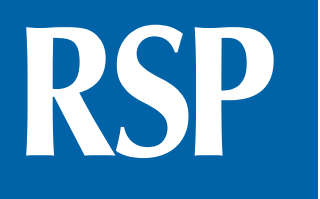

http://www.rsp.fsp.usp.br/
Revista de Saúde Pública

\title{
Tendência de mortalidade por hepatites B e C no município de São Paulo, 2002-2016
}

\author{
Ana Paula Sayuri Sato' iD, Inês Kazue Koizumi" iD, Norma Suely de Oliveira Farias" \\ Célia Regina Ciccolo da Silva"I' iD, Maria Regina Alves Cardoso' iD, Gerusa Maria Figueiredo ${ }^{\mathrm{IV}}$ \\ I Universidade de São Paulo. Faculdade de Saúde Pública. Departamento de Epidemiologia. São Paulo, SP, Brasil \\ " Secretaria de Estado da Saúde de São Paulo.Coordenadoria de Controle de Doenças. Centro de Vigilância \\ Epidemiológica "Prof. Alexandre Vranjac". São Paulo, SP, Brasil \\ III Secretaria Municipal de Saúde de São Paulo. Coordenadoria de Vigilância em Saúde. Divisão de Vigilância \\ Epidemiológica. Programa Municipal de Hepatites Virais. São Paulo, SP, Brasil \\ Iv Universidade de São Paulo. Faculdade de Medicina. Departamento de Medicina Preventiva. São Paulo, SP, \\ Brasil
}

\section{RESUMO}

OBJETIVO: Descrever a mortalidade por hepatites B e C como causa básica no município de São Paulo, verificando a tendência dessas taxas, e avaliar a associação dessas doenças a outras no período de 2002 a 2016.

MÉTODOS: Trata-se de um estudo de série temporal sobre mortalidade por hepatites B e C segundo sexo, com dados do Sistema de Informação de Mortalidade. A regressão de Prais Winsten foi usada na análise de tendência das taxas.

RESULTADOS: Os achados do presente estudo mostraram tendência de declínio da mortalidade por hepatites $\mathrm{B}$ e $\mathrm{C}$ nos últimos anos, particularmente entre pessoas do sexo masculino. Essas infecções foram causas associadas importantes ao carcinoma de células hepáticas e ao HIV. Destaca-se a proporção de óbitos com menos de 70 anos de idade.

Correspondência:

Ana Paula Sayuri Sato

Av. Dr. Arnaldo, 715,

01246-904, São Paulo, SP, Brasil

E-mail: sah@usp.br

Recebido: 28 nov 2019

Aprovado: 18 mar 2020

Como citar: Sato APS, Koizumi IK Farias NSO, Silva CRC, Cardoso MRA, Figueiredo GM. Tendência de mortalidade por hepatites B e C no município de São Paulo, 2002-2016. Rev Saude Publica. 2020;54:124.

Copyright: Este é um artigo de acesso aberto distribuído sob os termos da Licença de Atribuição Creative Commons, que permite uso irrestrito, distribuição e reprodução em qualquer meio, desde que o autor e a fonte originais sejam creditados.
CONCLUSÕES: O estudo fornece uma linha de base para pesquisas de tendência de mortalidade e de impacto de intervenções, visto o histórico de ampliação da detecção e oferta de tratamentos, incluindo os mais recentes antivirais no Brasil, desde 2015.

DESCRITORES: Hepatite B. Hepatite C. Mortalidade, tendências. Estudos de Séries Temporais. 


\section{INTRODUÇÃO}

As hepatites B e C apresentam grande magnitude no mundo, com estimativas de cerca de 257 milhões de pessoas cronicamente infectadas pelo vírus da primeira (VHB) e 71 milhões pelo vírus da segunda $(\mathrm{VHC})^{1}$. No Brasil, um inquérito populacional realizado nas capitais e no Distrito Federal na década de 2010, com pessoas de 10 a 69 anos, apresentou prevalência de 0,37\% e 7,40\% para o antígeno de superfície do vírus B (HBsAg) e contra o núcleo (Anti-HBc), respectivamente, e de 1,38\% para o anticorpo contra o vírus C (Anti-HCV) ${ }^{2-4}$. Isso resultou em uma estimativa para população residente nessas áreas de 430.658 pessoas Anti-HCV reagentes, sendo 36,70\% virêmicas ${ }^{4}$. Em 2016, o Ministério da Saúde estimou prevalência de $0,70 \%$ no país todo e de 657.000 pessoas com o vírus ativo da hepatite C, visando estabelecer uma linha de base para calcular os casos a diagnosticar e tratar anualmente, a fim de cumprir metas e eliminar as hepatites virais até $2030^{5}$. Em publicação mais recente essas estimativas foram atualizadas para 0,53\% e 632.000, respectivamente ${ }^{6}$. Essas informações mostram a importância dessas infecções no Brasil e o desafio para os serviços de saúde em detectar e tratar os portadores.

A hepatite crônica pelo VHB ou VHC tem sido associada ao aumento do risco de morte, sobretudo por causas relacionadas ao desenvolvimento de doenças hepáticas, como cirrose ou hepatocarcinoma (HCC). Em 2015, 887.000 pessoas morreram em consequência da infecção pelo $\mathrm{VHB}^{7}$, e em 2016, aproximadamente 399.000 morrem a cada ano com hepatite C, principalmente por cirrose e carcinoma hepatocelular8.

As hepatites virais estão entre as principais causas de morte em todo o mundo, sendo o HCC o único câncer que aumentou substancialmente entre os anos 1990 e 2013 ${ }^{9}$. No Brasil, a taxa de mortalidade por hepatite B no período de 2000 a 2009 foi de 0,3/100.000 habitantes-ano ${ }^{10}$.

Ressalte-se que os estudos sobre mortalidade por hepatite C no país são escassos ${ }^{5}$. No município de São Paulo, a tendência de mortalidade por hepatite B ou C não foi avaliada até o momento. Assim, o objetivo deste estudo é descrever a mortalidade por hepatites B e C como causa básica no município de São Paulo, verificando a tendência dessas taxas, e avaliar a participação dessas infecções como causas associadas a outras no período de 2002 a 2016.

\section{MÉTODOS}

Trata-se de estudo ecológico de séries temporais sobre a mortalidade por hepatites virais B e C, no período de 2002 a 2016, compreendendo óbitos de residentes no município de São Paulo.

A capital paulista tem área territorial de 1.522,986 km², população estimada em 11.638 .802 habitantes ${ }^{11}$ para 2016 e Índice de Desenvolvimento Humano Municipal de 0,805 ${ }^{12}$.

Utilizaram-se dados referentes aos óbitos no município ocorridos no período de 2002 a 2016, registrados no Sistema de Informações sobre Mortalidade (SIM) ${ }^{13}$. A fonte de dados foi o Programa de Aprimoramento das Informações de Mortalidade (PRO-AIM), gestor municipal do SIM e responsável por alimentar as informações, enviá-las à base federal e fazer a manutenção do sistema municipal. O PRO-AIM possui métodos para aprimorar as informações constantes nas declarações de óbito, com intuito de alcançar e manter a boa qualidade do sistema, como o envio de cartas aos médicos atestantes para o esclarecimento de dúvidas, investigação junto ao Instituto Médico Legal e ao Serviço de Verificação de Óbitos, entre outros ${ }^{14}$. Para o cálculo das taxas, foram utilizadas estimativas populacionais da Fundação Sistema Estadual de Análise de Dados (Seade) ${ }^{11}$.

Definiram-se como óbitos por hepatite B aqueles que apresentavam os seguintes códigos da Classificação Internacional de Doenças (CID-10) ${ }^{15}$ : B16.0 [hepatite aguda B com agente Delta (coinfecção) com coma hepático], B16.1 [hepatite aguda B com agente Delta (coinfecção) sem coma hepático], B16.2 (hepatite aguda B sem agente Delta, com coma hepático), B16.9 
(hepatite aguda B sem agente Delta, sem coma hepático), B17.0 (superinfecção Delta aguda de portador de hepatite crônica B), B18.0 (hepatite viral crônica B com agente Delta) e B18.1 (hepatite viral crônica B sem agente Delta). A definição de óbito por hepatite $\mathrm{C}$ compreendeu os códigos B17.1 (hepatite C aguda) ou B18.2 (hepatite C crônica) da CID-10 ${ }^{15}$.

A causa básica de morte é definida pela Organização Mundial da Saúde como “(a) a doença ou lesão que iniciou a sucessão de eventos patológicos que levou diretamente à morte, ou (b) as circunstâncias do acidente ou violência que produziu a lesão fatal". Causas associadas são todas as descritas na declaração de óbito diferentes da causa básica, ou seja, condições que contribuíram no processo de falecimento (contribuintes) ou que foram complicações da causa básica (consequenciais). Constituem causas múltiplas todas aquelas descritas na declaração de óbito, ou seja, as causas básicas e as associadas ${ }^{15,16}$.

Inicialmente consideraram-se apenas os óbitos cuja causa básica foi a hepatite B ou C, para o cálculo das taxas de mortalidade e para a análise de tendência. Em seguida, foi analisada a frequência com que as hepatites B e C estavam associadas a óbitos que tinham outra causa básica. Para isso, verificaram-se as causas associadas para definição de morte, ou seja, considerou-se a presença dos códigos da CID-10 ${ }^{15}$ citados anteriormente em qualquer linha da declaração de óbito ${ }^{17}$.

A taxa bruta de mortalidade foi calculada como o quociente entre o total de óbitos atribuídos à hepatite B ou C e a população na faixa etária estudada residente no município de São Paulo, em cada ano e multiplicado por 100.000. A padronização dos coeficientes de mortalidade foi realizada pelo método direto, utilizando como referência a população do censo de 2010 do município de São Paulo.

A análise de tendência foi realizada por meio de modelo de regressão linear para séries temporais com método de Prais-Winsten, a fim de minimizar a autocorrelação de primeira ordem dos resíduos. As variáveis dependentes foram as taxas padronizadas de mortalidade por hepatite $\mathrm{B}$ e C, e a variável independente foi o ano-calendário ${ }^{18}$. Utilizou-se o software Stata versão 15 para a análise estatística dos dados.

O projeto foi aprovado pelo Comitê de Ética da Secretaria Municipal de Saúde da Prefeitura da Cidade de São Paulo (Parecer n 510.009, de 6 de fevereiro de 2014).

\section{RESULTADOS}

\section{Hepatite B}

No período de 2002 a 2016, foram registrados no Sistema de Informação sobre Mortalidade do município de São Paulo 1.243 óbitos com menção à hepatite viral B, sendo 540 (43,44\%) codificados como causa básica e 703 (56,55\%) como causa associada.

Entre os 540 óbitos com hepatite B como causa básica, 127 (23,52\%) foram decorrentes de infecção aguda e 413 (76,48\%), crônica. Dentre os pacientes, 69,60\% eram do sexo masculino e $30,40 \%$, do feminino. A raça/cor branca foi predominante $(61,11 \%)$, e a maioria $(61,65 \%)$ tinha menos de 8 anos de escolaridade. A média da idade do sexo feminino foi de 66,00 anos, maior do que a do sexo masculino (58,61 anos). Cerca de dois terços dos óbitos foram de pessoas com menos de 70 anos de idade, e esta porcentagem é maior no sexo masculino, com 75,83\% (Tabela 1). Não foram registrados óbitos de pessoas abaixo de 15 anos de idade.

Quanto às causas associadas, foram encontrados 289 (53,52\%) óbitos com cirrose hepática, e 22 (4,07\%) com carcinoma hepatocelular (dados não apresentados em tabelas).

No período de 2002 a 2016, as taxas de mortalidade por hepatite B como causa básica variaram de 0,17 (2003 e 2006) a 0,58 (2013) por 100.000 habitantes-ano. Houve declínio 
anual de 5,10\% (IC95\%: -8,26; -1,82\%) em pessoas do sexo masculino (-4,75\%; IC95\%: -7,03; $-2,42 \%)$, mas não nas do feminino (-6,79\%; IC95\%: -14,10; 1,14\%) (Figura 1 e Tabela 2).

Encontrou-se menção à hepatite B em 703 óbitos cuja causa básica foram doenças constantes em capítulos da CID-10. A maior proporção está associada a doenças do Capítulo II, referente a neoplasias (C00-D48), com 42,53\% dos óbitos ( $\mathrm{n}=299)$, e ao Capítulo I, referente a doenças infecciosas e parasitárias (A00-B99), com 30,44\% ( $=214)$. Entre as neoplasias destacam-se

Tabela 1. Características dos óbitos por hepatites B e C como causas básicas, São Paulo, SP, 2002-2016.

\begin{tabular}{|c|c|c|c|c|}
\hline \multirow{2}{*}{ Variável } & \multicolumn{2}{|c|}{ Hepatite B $(n=540)$} & \multicolumn{2}{|c|}{ Hepatite C $(n=3194)$} \\
\hline & $\mathbf{n}$ & $\%$ & $\mathbf{n}$ & $\%$ \\
\hline \multicolumn{5}{|l|}{ Sexo } \\
\hline Feminino & 164 & 30,40 & 1433 & 44,90 \\
\hline Masculino & 376 & 69,60 & 1761 & 55,10 \\
\hline \multicolumn{5}{|l|}{ Faixa etária } \\
\hline $15-34$ & 20 & 3,71 & 45 & 1,30 \\
\hline $35-54$ & 164 & 30,43 & 885 & 27,80 \\
\hline $55-69$ & 191 & 35,43 & 1372 & 43,00 \\
\hline$\geq 70$ & 165 & 30,43 & 892 & 27,90 \\
\hline \multicolumn{5}{|l|}{ Raça/cor } \\
\hline Branca & 330 & 61,11 & 2428 & 76,02 \\
\hline Preta & 33 & 6,11 & 137 & 4,29 \\
\hline Parda & 105 & 19,44 & 400 & 12,52 \\
\hline Amarela & 29 & 5,37 & 56 & 1,75 \\
\hline Indígena & - & - & 1 & 0,03 \\
\hline Ignorada & 43 & 7,96 & 172 & 5,39 \\
\hline \multicolumn{5}{|l|}{ Escolaridade } \\
\hline Nenhuma & 35 & 6,48 & 169 & 5,29 \\
\hline $1-3$ & 104 & 19,26 & 652 & 20,41 \\
\hline $4-7$ & 115 & 21,30 & 741 & 23,20 \\
\hline $8-11$ & 99 & 18,33 & 657 & 20,57 \\
\hline$\geq 12$ & 59 & 10,93 & 416 & 13,02 \\
\hline Ignorada & 128 & 23,70 & 559 & 17,50 \\
\hline
\end{tabular}

Hepatite B

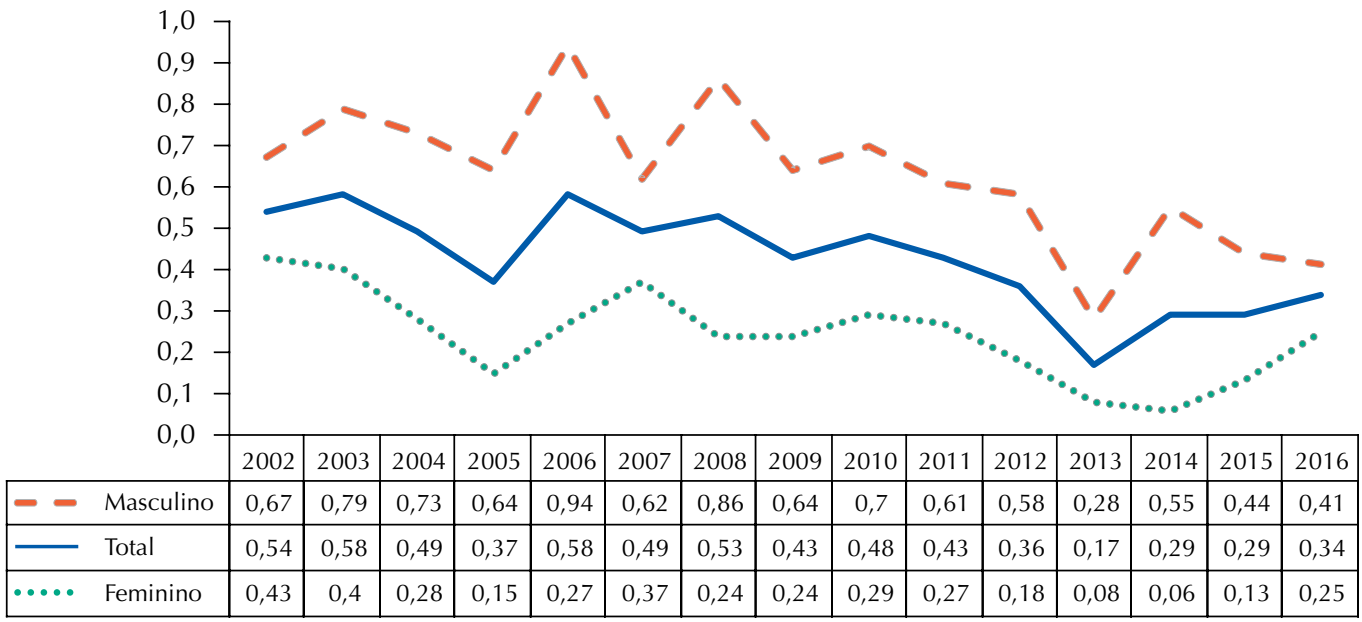

Fonte: SIM/PRO-AIM/CEINFO/SMSSP.

Figura 1. Taxas de mortalidade padronizadas por hepatite B como causa básica, segundo sexo e ano do óbito, São Paulo, SP, 2002-2016. 
o carcinoma de células hepáticas (C22.0) (56,18\%, 168/299) e a neoplasia maligna do fígado não especificada (C22.9), com 57 óbitos (19,06\%, 57/299). Entre as doenças infecciosas sobressaem-se as associadas ao HIV (B20.0, B20.1, B20.3, B20.6, B20.7, B20.8, B21.0, B22.7, B24), com 172 óbitos $(80,37 \%, 172 / 214)$. Dentre essas também se destacam 14 falecimentos cuja causa básica foi hepatite C (B17.1 e B18.2), (6,54\%, 14/214). No Capítulo XI - Doenças do Aparelho Digestivo, chamam atenção 28 óbitos por doenças relacionadas à ingestão de álcool (K70.0, K70.1, K70.3, K70.4 e K70.9) (dados não apresentados em tabelas).

\section{Hepatite C}

No período de 2002 a 2016 foram registrados 6.419 óbitos com menção à hepatite C, sendo $3.194(49,76 \%)$ como causa básica e 3.225 (50,24\%) como causa associada em residentes no município de São Paulo.

Entre as 3.194 pessoas falecidas com hepatite $\mathrm{C}$ como causa básica, $55,10 \%$ eram do sexo masculino e 44,90\% do feminino. A raça/cor branca constava em $76,02 \%$ dos registros, e $48,90 \%$ dos pacientes tinham menos de 8 anos de escolaridade. A média de idade foi de 61,72 anos, maior entre as mulheres (65,64 anos) em comparação aos homens (58,51 anos). Não foram registrados óbitos em menores de 15 anos de idade. Constatou-se que 72,12\% dos óbitos foram de pessoas com menos de 70 anos de idade, sendo esta porcentagem maior no sexo masculino, com 81,91 \% (Tabela 1).

Além disso, 1.801 óbitos $(56,41 \%)$ por hepatite C tinham cirrose hepática como causa associada. Destes casos, as mulheres (44,50\%) apresentaram média de idade de 66,22 anos e os homens (55,50\%), 57,93 anos. Identificaram-se 196 (6,14\%) óbitos com carcinoma hepatocelular como causa associada (dados não apresentados em tabelas).

As taxas de mortalidade por hepatite C como causa básica apresentaram tendência de aumento de 5,65\% ao ano (IC95\% 2,99;8,39\%) entre 2002 (1,99/100.000 habitantes-ano) e 2009 (3,07/100.000 habitantes-ano), tanto no sexo masculino (4,60\%; IC95\% 2,41;6,84\%) quanto no feminino (6,90\%; IC95\% 3,03;10,91\%). De 2009-2016, houve tendência de diminuição anual de 4,79\% (IC95\% -7,44; -2,07), passando para 1,98/100.000 habitantes em 2016. Esse declínio esteve presente em indivíduos do sexo masculino (-4,95\%; IC95\% -7,63; -2,19\%), mas não do feminino (-5,29\%; IC95\% -10,98; 0,76\%) (Figura 2 e Tabela 2).

Em relação aos 3.225 óbitos com menção à hepatite pelo vírus $\mathrm{C}$ como causa associada, a maior proporção tinha como causa básica as neoplasias (Capítulo II do CID-10, C00-D48, $\mathrm{n}=1.246,38,63 \%$ ), seguidas de doenças infecciosas e parasitárias (Capítulo I do CID-10,

Hepatite C

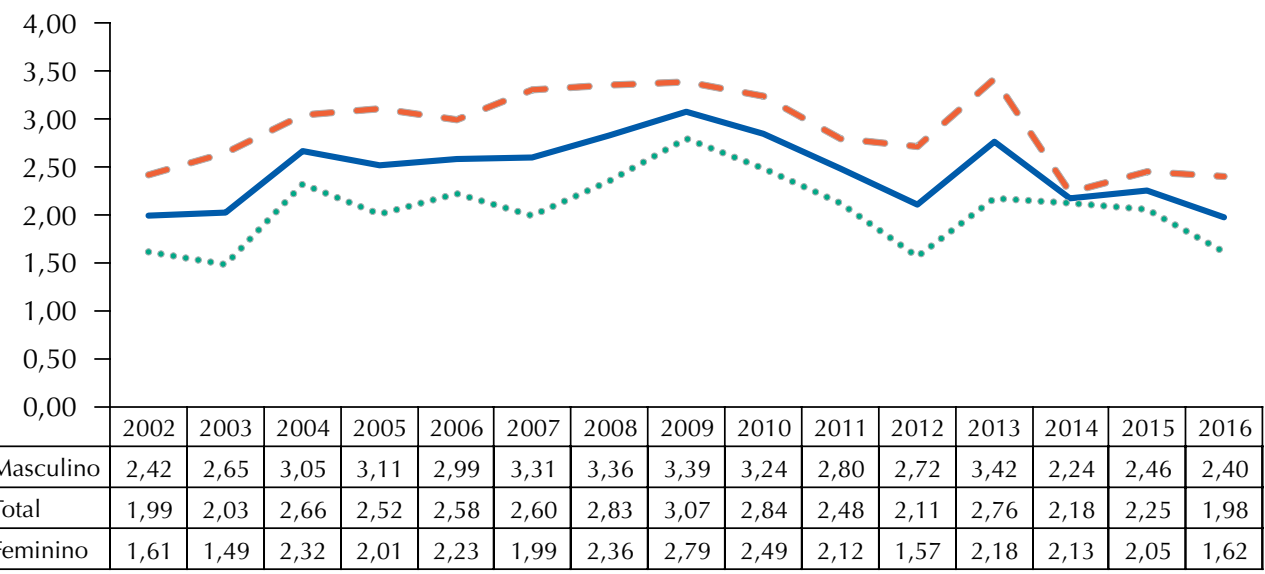

Fonte: SIM/PRO-AIM/CEINFO/SMSSP.

Figura 2. Taxas de mortalidade padronizadas por hepatite C como causa básica, segundo sexo e ano do óbito, São Paulo, SP, 2002-2016. 
Tabela 2. Variação anual média das taxas de mortalidade por hepatites B e C como causas básicas, segundo sexo e período estudado, São Paulo, SP, 2002-2016.

\begin{tabular}{lcccc}
\hline \multirow{2}{*}{ Tipo/período } & Sexo & $\begin{array}{c}\text { Variação anual média } \\
(\%)\end{array}$ & \multicolumn{2}{c}{ Intervalo de confiança de 95\% } \\
\cline { 4 - 5 } Hepatite B & & & - & + \\
\hline $2002-2016$ & Geral & $-5,10$ & $-8,26$ & $-1,82$ \\
& Masculino & $-4,75$ & $-7,03$ & $-2,42$ \\
\hline Hepatite C & Feminino & $-6,79$ & $-14,10$ & 1,14 \\
$2002-2009$ & Geral & & & \\
& Masculino & 5,65 & 2,99 & 8,39 \\
\hline $2009-2016$ & Feminino & 4,60 & 2,41 & 6,84 \\
& Geral & 6,90 & 3,03 & 10,91 \\
& Masculino & $-4,79$ & $-7,44$ & $-2,07$ \\
& Feminino & $-4,95$ & $-7,63$ & $-2,19$ \\
\hline
\end{tabular}

A00-B99, $\mathrm{n}=893,27,69 \%)$. Entre as neoplasias, o carcinoma de células hepáticas (C22.0) correspondeu a 59,95\% dos óbitos (747/1.246), seguido de neoplasia maligna do fígado não especificada (C22.9) $(14,77 \%, 184 / 1.246)$. Entre as doenças infecciosas, 78,61\% dos óbitos (702/893) tinham doenças associadas ao HIV como causa básica (B20.0, B20.1, B20.2, B20.3, B20.6, B20.7, B20.8, B21.0, B21.1, B21.7, B21.8, B22.0, B22.7, B23.2, B24), e 7,61\% (68/893) tinham a hepatite B (B16.2, B16.9, B18.1). Destaca-se que 113 indivíduos faleceram por doenças hepáticas relacionadas à ingestão de álcool (K70.0, K70.1, K70.3, K70.4 e K70.9) (dados não apresentados em tabelas).

\section{DISCUSSÃO}

Os achados do presente estudo mostraram tendência de declínio da mortalidade por hepatites B e C nos últimos anos no município de São Paulo, particularmente no sexo masculino. Essas infecções foram importantes causas associadas ao carcinoma de células hepáticas e ao HIV. Além disso, destaca-se a proporção de indivíduos que faleceu com menos de 70 anos de idade (cerca de $70 \%$ ).

A análise de tendência da mortalidade por hepatite B mostrou redução, particularmente em homens. Nos EUA, a tendência da mortalidade por hepatite B entre 1999 e 2007 mostrou que a média anual ajustada por idade foi relativamente constante ${ }^{19}$. Mas em outros locais, como Taiwan, região hiper endêmica em décadas passadas, teve quedas acentuadas , $^{4,221}$. Sugere-se que a diminuição das taxas de mortalidade por hepatite B no Brasil possa ser atribuída em parte à vacinação, com diminuição da prevalência, mas também às medidas de prevenção contra a infecção pelo HIV iniciadas na década de 1980, como observado em outros países ${ }^{4,22,23}$.

Em relação à mortalidade por hepatite $\mathrm{C}$, verificou-se tendência de aumento médio de 5,65\% ao ano entre 2002 e 2009, em ambos os sexos, e de redução de 4,79\% ao ano de 2009 a 2016, no sexo masculino. Nos EUA, a taxa de mortalidade anual padronizada apresentou aumento e ultrapassou a mortalidade por aids, entre 1999 e $2007^{19}$. No Brasil, chama atenção a queda percentual no segundo período, que em parte pode ser devida ao tratamento instituído no país no início da década anterior, com terapia dupla, utilizada até 2014, incluindo interferon peguilado como um de seus componentes, além do aumento de captação pelos serviços de pessoas infectadas ${ }^{24-26}$. Em 2015, o Ministério da Saúde instituiu o tratamento com novos antivirais de ação direta, que mostram eficácia de mais de $90 \%$ na cura da hepatite $\mathrm{C}$ e menores índices de efeitos adversos ${ }^{27}$. 
O cuidado clínico para o paciente com HCC tem avançado consideravelmente, devido à evolução dos procedimentos diagnósticos e à melhoria na terapia e prevenção. $\mathrm{O}$ tratamento da infecção vem sendo proposto e aperfeiçoado há cerca de 20 anos, com um arsenal terapêutico de eficácia progressivamente melhor.

Em muitos países, a incidência das hepatites $\mathrm{B}$ e $\mathrm{C}$ diminuiu devido ao menor número de novas infecções. No entanto, a prevalência de doenças hepáticas graves continua aumentando, pois existe um contingente de pessoas infectadas pelo VHB e pelo VHC em décadas passadas que desconhecem sua condição ou que foram diagnosticadas tardiamente, quando apresentaram sinais e sintomas de doença hepática grave, como cirrose e HCC, o que contribui para a morte prematura ${ }^{9,28}$.

Nos EUA, um estudo de coorte, de 2006 a 2010, mostrou média de idade de 57 anos para os óbitos por hepatite $\mathrm{C}^{29}$. Outro estudo conduzido na cidade de Nova York, com casuística de 2000 a 2011, apontou que 64,10\% dos óbitos de indivíduos infectados pelo VHC ocorreram antes de 65 anos de idade ${ }^{30}$.

Neste estudo, observou-se que pelo menos dois terços dos óbitos por hepatites B e C acometeram indivíduos com idade inferior a 70 anos, ou seja, os nascidos entre 1945 e 1965 (“baby boomers"). No caso da hepatite C, essas pessoas se infectaram principalmente antes da incorporação da triagem em bancos de sangue e de novas tecnologias de produtos e processos relacionados ao controle de sangue e hemoderivados, bem como normas de biossegurança em serviços de saúde ${ }^{31}$. Esse achado fortalece a recomendação para testagem Anti-HCV em pessoas nascidas nesse período, visto que a infecção pode ser assintomática em $75 \%$ dos $\operatorname{casos}^{32}$, bem como para a instituição do tratamento precoce e a vacinação contra o VHB nesses portadores, conforme protocolo preconizado, impedindo o agravamento do seu quadro ${ }^{33}$.

A significativa proporção de hepatites B e C contribuindo como causas associadas ao HCC e ao HIV é consistente com outros achados na literatura. Uma revisão sistemática de estudos publicados em diversos países mostrou que as hepatites virais crônicas pelo VHB e VHC contribuem para a maioria das ocorrências de $\mathrm{HCC}^{33}$. Globalmente, 78\% das infecções por HCC foram atribuíveis ao HBV (53\%) e HCV (25\%), confirmando que as hepatites crônicas B e C representam a principal causa de câncer primário de fígado no mundo ${ }^{7-8,33}$. A coinfecção das hepatites virais com o HIV pode ser explicada pelas vias comuns de infecção, principalmente a parenteral e sexual ${ }^{34}$, e está associada ao maior risco de morte ${ }^{27,35}$. A progressão da fibrose hepática para cirrose é acelerada em indivíduos com hepatite $\mathrm{C}$ e HIV concomitantes ${ }^{36}$.

Os dados de mortalidade do presente estudo podem estar subestimados devido à ausência da hepatite B ou C na declaração de óbito, seja como causa básica ou associada ${ }^{28}$. Estudaram-se também as causas associadas de óbito para tentar entender o efeito do sub-registro das hepatites virais $\mathrm{B}$ e $\mathrm{C}$ como causa básica, principalmente quando associadas à cirrose hepática, HCC e infecção pelo vírus HIV ${ }^{37}$.

As estatísticas de mortalidade segundo causa básica deixam de mostrar o impacto total de uma doença ou agravo, em um conjunto de óbitos ${ }^{38}$. O presente estudo possibilitou esta visão mais ampla e completa, evidenciando a presença das hepatites virais B e C como causas associadas, de forma a contribuir para o conhecimento do perfil epidemiológico desses agravos em nosso meio.

Os dados encontrados mostram a importância da prevenção e do diagnóstico precoce e tratamento das hepatites B e C. A contribuição desse trabalho é fornecer uma linha de base para novos estudos de tendência de mortalidade e de avaliação do impacto de novas intervenções, considerando a ampliação do diagnóstico e da oferta de tratamentos, incluindo os mais recentes antivirais no Brasil, desde $2015^{27}$, sendo independente do grau de fibrose hepática, desde $2019^{39}$. Além disso, esses resultados podem subsidiar o alcance de metas previstas para a eliminação das hepatites B e C no município de São Paulo ${ }^{39,40}$. 


\section{REFERÊNCIAS}

1. World Health Organization. Prevention and control of viral hepatitis infection: framework for global action. Geneva: WHO; 2012.

2. Pereira LMMB, Martelli CMT, Méchan-Hamann E, Montarroyos UR, Braga MC, Lima MLC, et al. Population-based multicentric survey of hepatitis B infection and risk factor differences among three regions in Brazil. Am J Trop Med Hyg. 2009;81(2):240-7. https://doi.org/10.4269/ajtmh.2009.81.240

3. Pereira LMMB, Martelli CMT, Moreira RC, Merchan-Hamman E, Stein AT, Cardoso MRA, et al. Prevalence and risk factors of Hepatitis C virus infection in Brazil, 2005 through 2009: a cross-sectional study. BMC Infect Dis. 2013;13:60. https://doi.org/10.1186/1471-2334-13-60

4. Ximenes RAA, Figueiredo GM, Cardoso MRA, Stein AT, Moreira RC, Coral G, et al. Population-based multicentric survey of Hepatitis B infection and risk factors in the north, south, and southeast regions of Brazil, 10-20 years after the beginning of vaccination. Am J Trop Med Hyg. 2015;93(6):1341-8. https://doi.org/10.4269/ajtmh.15-0216

5. Ministério da Saúde (BR), Secretaria de Vigilância em Saúde, Departamento de DST/aids e Hepatites Virais. Plano para Eliminação da Hepatite C no Brasil. Brasília, DF; 2019.

6. Benzaken AS, Girade R, Catapan E, Pereira GFM, Almeida EC, Vivaldini S, et al. Hepatitis C disease burden and strategies for elimination by 2030 in Brazil. A mathematical modeling approach. Braz J Infect Dis. 2019;23(3):182-90. https://doi.org/10.1016/j.bjid.2019.04.010

7. World Health Organization. Hepatitis B. Geneva: WHO; 2020 [citado 9 set 2020]. Disponível em : https://www.who.int/news-room/fact-sheets/detail/hepatitis-b

8. World Health Organization. Hepatitis C. WHO; Geneva; WHO; 2020 [citado 9 set 2020]. Disponível em: https://www.who.int/news-room/fact-sheets/detail/hepatitis-c

9. GBD 2016 Causes of Death Collaborators. Global, regional, and national age-sex specific mortality for 264 causes of death, 1980-2016: a systematic analysis for the Global Burden of Disease Study 2016. Lancet. 2017;390(10100):1151-1210. https://doi.org/10.1016/S0140-6736(17)32152-9

10. Tauil MC, Amorim TR, Pereira GFM, Araújo WN. Mortalidade por hepatite viral B no Brasil, 2000-2009. Cad Saude Publica. 2012;28(3):472-8. https://doi.org/10.1590/S0102-311X2012000300007

11. Fundação SEADE. Informações dos Municípios Paulistas. São Paulo; 2019 [citado 1 nov 2019]. Disponível em: http://www.imp.seade.gov.br/frontend/\#/

12. Instituo Brasileiro de Geografia e Estatística. Índice de Desenvolvimento Humano Municipal 2010. Rio de Janeiro: IBGE; 2010 [citado 18 nov 2019]. Disponível em: https://cidades.ibge.gov. br/brasil/sp/sao-paulo/panorama

13. Ministério da Saúde (BR). DATASUS. Arquivo de dados do Sistema de Informação de Mortalidade do Estado de São Paulo do período de 2002 a 2016. Brasília, DF; 2019 [citado 1 nov 2019]. Disponível em: http://www2.datasus.gov.br/DATASUS/index. php?area $=0901$ \&item $=1$ \&acao $=26$

14. Prefeitura Municipal de São Paulo. Programa de Aprimoramento das Informações de Mortalidade. Coletânea de Textos dos boletins do PRO-AIM. São Paulo; 2003.

15. Organização Mundial da Saúde. CID-10. Classificação estatística internacional de doenças e problemas relacionados à saúde. 2.ed. São Paulo: Editora da Universidade de São Paulo;1997. v.1.

16. Laurenti R, Mello Jorge MHP. Atestado de óbito: aspectos médicos, estatísticos, éticos e jurídicos. São Paulo: Conselho Regional de Medicina do Estado de São Paulo; 2015.

17. Ministério da Saúde (BR), Fundação Nacional da Saúde. Manual de Instruções para o preenchimento da declaração de óbito. 3. ed. Brasília, DF: FUNASA; 2001.

18. Antunes JLF, Cardoso MRA. Uso da análise de séries temporais em estudos epidemiológicos. Epidemiol Serv Saude. 2015;24(3):565-76. https://doi.org/10.5123/S1679-49742015000300024

19. Ly KN, Xing J, Klevens RM, Jiles RB, Ward JW, Holmberg SD. The increasing burden of mortality from viral hepatitis in the United States between 1999 and 2007. Ann Intern Med. 2012;156(4):271-8. https://doi.org/10.7326/0003-4819-156-4-201202210-00004

20. Chang MH, Chen CJ, Lai MS, Hsu HM, Wu TC, Kong MS, et al. Universal hepatitis B vaccination in Taiwan and the incidence of hepatocellular carcinoma in children. N Engl J Med. 1997;336(26):1855-9. https://doi.org/10.1056/NEJM19970626336260 
21. Ni YH, Chang MH, Huang LM, Chen HL, Hsu HY, Chiu TY, et al. Hepatitis B virus infection in children and adolescents in a hyperendemic area: 15 years after mass hepatitis B vaccination. Ann Intern Med. 2001;135(9):796-800. https://doi.org/10.7326/0003-4819-135-9-200111060-00009

22. Grirnsven GJ, Vroome EM, Goudsmit J, Coutinho RA. Changes in sexual behavior and the fall in incidence of HIV infection among homosexual men. BMJ. 1989;298(6668):218-21. https://doi.org/10.1136/bmj.298.6668.218

23. Alter MJ, Hadler SC, Margolis HS, Alexander J, Hu PY, Judson FN, et al. The changing epidemiology of hepatitis $b$ in the United States. Needs for alternative vaccination strategies. JAMA. 1990;263(9):1218-22. https://doi.org/10.1001/jama.1990.03440090052025

24. Marotta P, Hueppe D, Zehnter E, Kwo P, Jacobson I. Efficacy of chronic hepatitis C therapy in community-based trials. Clin Gastroenterol Hepatol. 2009;7(10):1028-36. https://doi.org/10.1016/j.cgh.2009.05.003

25. Hansen N, Obel N, Christensen PB, Kjaer M, Laursen AL, Krarup HB, et al. Effectiveness of treatment with pegylated interferon and ribavirin in an unselected population of patients with chronic hepatitis C: a Danish nationwide cohort study. BMC Infect Dis. 2011;11:177. https://doi.org/10.1186/1471-2334-11-177

26. Ford N, Kirby C, Singh K, Mills EJ, Cooke G, Kamarulzaman A, et al. Chronic hepatitis $\mathrm{C}$ treatment outcomes in low- and middle-income countries: a systematic review and meta-analysis. Bull World Health Organ. 2012;90(7):540-50. https://doi.org/10.2471/BLT.11.097147

27. Ministério da Saúde (BR), Secretaria de Vigilância em Saúde. Departamento de DST, Aids e Hepatites Virais. Protocolo clínico e diretrizes terapêuticas para Hepatite C e coinfecções. Brasília, DF; 2015.

28. Razavi H, Elkhoury AC, Elbasha E, Estes C, Pasini K, Poynard T, et al. Chronic hepatitis C virus (HCV) disease burden and cost in the United States. Hepatology. 2013;57(6):2164-70. https://doi.org/10.1002/hep.26218

29. Mahajan R, Xing J, Liu SJ, LyKN, Moorman AC, Rupp L, et al. Mortality among persons in care with hepatitis C virus infection: the Chronic Hepatitis Cohort Study (CheCS), 2006-2010. Clin Infect Dis. 2014;58(8):1055-61 https://doi.org/10.1093/cid/ciu077

30. Pinchoff J, Drobnik A, Bornschlegel K, Braunstein S, Chan C, Varma JK et al. Deaths among people with hepatitis C in New York City, 2000-2011. Clin Infect Dis. 2014;58(8):1047-54. https://doi.org/10.1093/cid/ciu075

31. Ministério da Saúde (BR), Agência Nacional de Vigilância Sanitária. Portaria № 4, de 7 de fevereiro de 1986. Normaliza o uso e reutilização de materiais médico-hospitalares descartáveis no país. Diário Oficial União. 12 fev 1986;(28).

32. Ly KN, Xing J, Klevens RM, Jiles RB, Holmberg SD. Causes of death and characteristics of decedents with viral hepatitis, United States, 2010. Clin Infect Dis. 2014;58(1):40-9. https://doi.org/10.1093/cid/cit642

33. Perz JF, Armstrong GL, Farrington LA, Hutin YJF, Bell BP. The contributions of hepatitis B virus and hepatitis $C$ virus infections to cirrhosis and primary liver cancer worldwide. J Hepatol. 2006;45(4):529-38. https://doi.org/10.1016/j.jhep.2006.05.013

34. Sulkowski MS. Viral hepatitis and HIV coinfection. J Hepatol. 2008;48(2):353-67. https://doi.org/10.1016/j.jhep.2007.11.009

35. Walter SR, Thein HH, Amin J, Gidding HF, Ward K, Law MG, et al. Trends in mortality after diagnosis of hepatitis B or C infection: 1992-2006. J Hepatol. 2011;54(5):879-86. https://doi.org/10.1016/j.jhep.2010.08.035

36. Lédinghen V, Barreiro P, Foucher J, Labarga P, Castéra L, Vispo $M E$, et al. Liver fibrosis on account of chronic hepatitis $C$ is more severe in HIV-positive than HIV-negative patients despite antiretroviral therapy. J Viral Hepat. 2008;15(6):427-33. https://doi.org/10.1111/j.1365-2893.2007.00962.x

37. Redelings MD, Sorvillo F, Simon P. A comparison of underlying cause and multiple causes of death. Epidemiology. 2006;17(1):100-3. https://doi.org/10.1097/01.ede.0000187177.96138.c6

38. Oliveira BZ, Gotlieb SLD, Laurenti R, Mello Jorge MHP. Mortalidade feminina por hipertensão: análise por causas múltiplas. Rev Bras Epidemiol. 2009;12(4):556-65. https://doi.og/10.1590/S1415-790X2009000400006 
39. Ministério da Saúde (BR), Secretaria de Vigilância em Saúde. Departamento de Vigilância, Prevenção e Controle das Infecções Sexualmente Transmissíveis, do HIV/Aids e das Hepatites Virais. Protocolo clínico e diretrizes terapêuticas para Hepatite C e coinfecções; Brasília, DF; 2019.

40. Kabiri M, Jazwinski AB, Roberts MS, Schaefer AJ, Chhatwal J. The changing burden of hepatitis $C$ virus infection in the United States: model-based predictions. Ann Intern Med. 2014;161(3):170-80. https://doi.org/10.7326/M14-0095

Contribuição dos Autores: Concepção e planejamento do estudo: APSS, IKK, MRAC, NSOF, CRCS. Coleta, análise e interpretação dos dados: APSS, IKK, MRAC, GMF. Elaboração ou revisão do manuscrito: APSS, IKK, NSOF, CRCS, MRAC, GMF. Aprovação da versão final: APSS, IKK, NSOF, CRCS, MRAC, GMF. Responsabilidade pública pelo conteúdo do artigo: APSS, IKK, NSOF, CRCS, MRAC, GMF.

Conflito de Interesses: Os autores declaram não haver conflito de interesses. 